\title{
Development of a Gold Nanoparticle-Functionalized Surface Plasmon Resonance Assay for the Sensitive Detection of Monoclonal Antibodies and Its Application in Pharmacokinetics ${ }^{\mathbb{S}}$
}

\author{
Haihong Bai, Mei Yuan, Xiaojing Wang, Xinghe Wang, ${ }^{1}$ and Jinjing Che ${ }^{1}$
}

Phase I Clinical Trial Center, Beijing Shijitan Hospital of Capital Medical University, Beijing, PR China (H.B., Xin.W.); State Key Laboratory of Toxicology and Medical Countermeasures, Beijing Institute of Pharmacology and Toxicology, Beijing, PR China (M.Y., J.C.); and Chinese Pharmaceutical Association, Beijing, PR China (Xia.W.)

Received February 9, 2019; accepted July 15, 2019

\section{ABSTRACT}

As a prominent human therapeutic, therapeutic monoclonal antibodies (mAbs) have attracted increasing attention in the past decade due to their high-targeting specificity, low toxicity, and prolonged efficacy. Systematic pharmacokinetic analysis of mAbs not only largely facilitates the understanding of their biologic functions but also promotes the development of therapeutic drug discovery, early clinical trial implementation, and therapeutic monitoring. However, the extremely complex nature of biomatrices and the especially low dosages of mAbs make their detection in biomatrices and further pharmacokinetic analysis highly challenging. Therefore, a method capable of reliably, quickly, and sensitively quantifying mAbs in biomatrices is urgently needed. In this work, we developed and evaluated an gold nanoparticle-functionalized surface plasmon resonance assay for cetuximab (C225) detection and pharmacokinetic analysis in rhesus monkeys. Combining its advantages of label-free pretreatment and amplified signal response, the lower limit of quantitation of C225 in monkey serum was reduced to $0.0125 \mu \mathrm{g} / \mathrm{ml}$, and the linear range had an order of magnitude comparable to that of an ELISA-based method. Furthermore, the pharmacokinetics of $\mathrm{C225}$ in rhesus monkeys was studied after intravenous infusions of single doses at 7.5, 24, and $75 \mathrm{mg} / \mathrm{kg}$. The concentration of $\mathrm{C225}$ in monkey serum was detectable after dosing for 720 hours. We believe that this new strategy will be applicable as a general protocol for mAb quantification, pharmacokinetic characteristic determination, and toxicokinetic analysis during drug development.

\section{Introduction}

Currently, nearly 350 monoclonal antibodies (mAbs) are being investigated in clinical trials of different stages (Wang et al., 2016; Singh et al., 2018). Despite remarkable advances, the absorption, distribution, metabolism, and elimination of mAbs, early clinical trial development, and therapeutic monitoring have not been completely elucidated. For example, considerable controversy exists regarding whether the Fc receptor is the main factor for tuning mAb pharmacokinetics (Gurbaxani et al., 2013). The pharmacokinetic of IgG molecules is complex, involving protective and clearance pathways related to neonatal Fc receptor-dependent recycling, target-mediated drug disposition, nonspecific or off-target binding-mediated clearance, and sometimes immunogenicity (Piche-Nicholas et al., 2018). To obtain parameters to further elucidate the pharmacokinetic, toxicokinetic, and clinical properties of mAbs, a method capable of reliable, fast, and

This work was supported by the National Natural Science Foundation of People's Republic of China [Grants 30873113 and 81603183] and the National Major Scientific and Technological Special Project for Significant New Drugs Development [Grant 2018ZX09J18102].

${ }^{1}$ Xin.W. and J.C. contributed equally to this work.

https://doi.org/10.1124/dmd.119.086249.

S This article has supplemental material available at dmd.aspetjournals.org. sensitive mAb quantification is urgently needed (Fischer et al., 2012; Li et al., 2016; Hage et al., 2018).

ELISA is currently one of the most prominent platforms for $\mathrm{mAb}$ quantification (Wolf et al., 2011; Salomon and Singh, 2015; Passot et al., 2017). ELISA pretreatment includes coating capture antibody, washing, and addition of sample, detection antibody, 3,3',5,5' -tetramethylbenzidine (TMB), and stop solution. This process generally takes 12 to 16 hours. The time-consuming ELISA pretreatment steps are major disadvantages and disrupt the fast and sensitive monitoring of mAbs in real time from early to late stages of drug development (Neill et al., 2015; Legeron et al., 2017). Surface plasmon resonance (SPR) is based on the principle that variation in the concentration of molecules in the solution may alter the refractive index of the solution close to the senor surface (Hamilton et al., 2012; Then et al., 2017; Jung et al., 2018). Biomolecular interaction analysis from Biacore using the optical SPR phenomenon can measure biomolecules in real time without requiring labeling or pretreatment (Fee, 2013; Olaru et al., 2015). Our group first reported a method for the quantification of $\mathrm{C} 255$ (cetuximab), a mAb (IgG1) directed at the extracellular domain of the receptor and further pharmacokinetic analysis of monkey serum using the Biacore system (Che et al., 2009). Although automated and accurate analysis was achieved, the relatively low detection sensitivity remained a major disadvantage of the method. The high-throughput detection and precise pharmacokinetic analysis of mAbs in complex biologic samples are prevented because mAbs generally exist in extremely low amounts and remain undetectable at specific time points after dosing (Li et al., 2012).

ABBREVIATIONS: Au NP, gold NP; C225, cetuximab; EGFR, epidermal growth factor receptor; LLOQ, lower limit of quantitation; mAb, monoclonal antibody; NP, nanoparticle; QC, quality control; SPR, surface plasmon resonance; $t_{1 / 2}$, half-life; TMB, 3,3',5,5'-tetramethylbenzidine. 
Various methods have been developed to enhance the sensitivity of SPR biosensors (Jazayeri et al., 2016; Chen et al., 2018; Hong et al., 2018; Hossain and Maragos, 2018). One of the frequently adopted procedures is to improve the signal intensity of the analyst by changing the refractive index at the sensor surface by introducing other chemical or nanoparticle (NP) agents, including secondary antibodies, antibody-enzyme conjugates, and metal NPs (Jazayeri et al., 2016; Chen et al., 2018; Hossain and Maragos, 2018). Gold NPs (Au NPs) have been widely used to amplify the responses of SPR biosensors due to the large shift in the plasmon angle, the broadened plasmon resonance, and the extremely high biocompatibility. Zhang et al. (2016) enhanced the bipyramidal Au $\mathrm{NP}$-containing sensor compared with the NP-free system in the labelfree detection of the analyte IgG. Ben Haddada et al. (2017) developed a stable $\mathrm{Au} \mathrm{NP}$ bioconjugate for the detection of staphylococcal enterotoxin A using localized SPR, and the limit of detection was estimated to be $5 \mathrm{ng} / \mathrm{ml}$, which was substantially lower than that obtained using a quartz crystal microbalance. Few reports on mAb quantification in complex biomatrices for pharmacokinetic analysis using $\mathrm{Au}$ NP-functionalized SPR exist to the best of our knowledge.

We elucidated the preclinical pharmacokinetic parameters of cetuximab (C225) in rhesus monkeys after intravenous infusion for the first time using a SPR biosensor-based method (Che et al., 2009). However, the lower limit of quantification (LLOQ) of the method was $0.05 \mu \mathrm{g} / \mathrm{ml}$. In this study, we further developed and evaluated an Au NPfunctionalized SPR assay for C225 detection and pharmacokinetic analysis in rhesus monkeys. Combining the advantages of label-free pretreatment and amplified signal response, the LLOQ was lowered to $0.0125 \mu \mathrm{g} / \mathrm{ml}$, and the linear range had an order of magnitude comparable to that of an ELISA-based method. Therefore, the concentration of $\mathrm{C} 225$ in monkey serum after intravenous injection for longer time was detectable. Furthermore, accurate pharmacokinetic parameters of $\mathrm{C} 225$ in rhesus monkeys were obtained. As a result, we believe that this new strategy will be applicable as a general protocol for $\mathrm{mAb}$ quantification, elucidating pharmacokinetic and toxicokinetic analysis during drug development.

\section{Materials and Methods}

Chemicals and Reagents. C225, Erbitux, and epidermal growth factor receptor (EGFR) were provided by Huabei Pharmaceutical Factory (Hebei Province, China). Goat anti-human $\mathrm{IgG}$ and bovine serum albumin were purchased from Sigma-Aldrich (St. Louis, MO). Proclin 300, TMB, and 3-[(3-cholamidopropyl)dimethyl-ammonio]-1-propane sulfonate were purchased from Sigma-Aldrich. Horseradish peroxidase-conjugated anti-goat IgG antibody was acquired from Bethyl (Montgomery, TX). The Biacore T200 biosensor, amine-coupling kit, CM5 sensor chip, $10 \mathrm{mM}$ acetate, $50 \mathrm{mM}$ $\mathrm{NaOH}$, glycine- $\mathrm{HCl}$ ( $\mathrm{pH} 2.0$ ), ethanolamine, and ethanolamine- $\mathrm{HCl}$ were purchased from Biacore AB (Uppsala, Sweden). N-hydroxysuccinimide and $\mathrm{N}$-ethyl- $\mathrm{N}^{\prime}$-(3-diethylaminopropyl) carbodiimide were purchased from Pierce (Rockford, IL). The deionized water (with resistance $>18 \mathrm{M} \Omega . \mathrm{cm}$ ) used in this experiment was prepared using a Millipore purification system (Billerica, MA).

Synthesis of Goat Anti-Human IgG-Au NP Conjugates. Briefly, 0.75, 1, and $3 \mathrm{ml} 1 \%$ trisodium citrate solution was added to $100 \mathrm{ml} 0.01 \% \mathrm{HAuCl}_{4}$ solution, and the mixture was allowed to react at $100^{\circ} \mathrm{C}$ for 5 to 30 minutes with vigorous stirring to obtain the Au NPs with the desired diameters.

Then, $20 \mathrm{ml} 15 \mu \mathrm{g} / \mathrm{ml}$ goat anti-human IgG (pH 9.0) was added to $20 \mathrm{ml}$ obtained Au NP solution ( $\mathrm{pH} 9.0$ ), and the mixture was stirred for 10 minutes at ambient temperature. Finally, $200 \mathrm{mg}$ bovine serum albumin was added, and the solution was centrifuged at $12,000 \mathrm{~g}$ for 60 minutes to remove any unbound molecules. The obtained goat anti-human IgG-Au NP conjugates were stored at $4^{\circ} \mathrm{C}$ for further use.

Au NP-Functionalized SPR Assay for C225 Quantification in Monkey Serum. All SPR measurements were performed on a Biacore T200 instrument (Biacore AB) using HBS-EP buffer [0.01 M HEPES (pH 7.4), $0.15 \mathrm{M} \mathrm{NaCl}$,
3 mM EDTA, $0.005 \%$ Tween 20 ] as the running buffer at a flow rate of $10 \mu \mathrm{l} / \mathrm{min}$. The Fc1 of the CM 5 sensor chip was used for EGFR immobilization using the amine-coupling kit according to the manufacturer's protocol, and $\mathrm{Fc} 2$ served as the reference. Next, $30 \mu \mathrm{l}$ EGFR $[100 \mu \mathrm{g} / \mathrm{ml}$ dissolved in $10 \mathrm{mM} \mathrm{NaOAc}$ (pH 4.5)] was injected and allowed to covalently couple to the sensor surface. Then the unreacted sites were blocked by the injection of $35 \mu \mathrm{l} 1 \mathrm{M}$ ethanolamine (pH 8.5). For quantitative analysis, $20 \mu \mathrm{l} \mathrm{C225}$ at different concentrations in $20 \%$ monkey serum and $60 \mu \mathrm{l}$ goat anti-human IgG-Au NP conjugates were injected and allowed to flow over the immobilized EGFR sensor surface and the reference flow cell at room temperature sequentially. The unbound goat antihuman IgG-Au NPs were removed by HBS-EP buffer. Data from the reference flow cell were subtracted to correct for nonspecific binding. The sensor surface was regenerated by injecting $10 \mu 110 \mathrm{mM} \mathrm{HCl}(\mathrm{pH} 2.0)$, followed by a return to HBS-EP buffer.

Method Validation. The established method was validated for a calibration curve, sensitivity, matrix effects, precision, accuracy, and stability, according to the Food and Drug Administration guidelines for bioanalytical method validation. The calibration curve for $\mathrm{C} 225$ ranging from 0.0125 to $3.2 \mu \mathrm{g} / \mathrm{ml}$ in $20 \%$ monkey serum was generated by plotting the Biacore response of the analyte versus the nominal concentration and fitted by a four-parameter sigmoidal model, as described in our previous work (Che et al., 2009). C225 at concentrations of $0.025,0.4$, and $1.6 \mu \mathrm{g} / \mathrm{ml}$ were set as the low, middle, and high quality control (QC) samples, respectively. The three QC samples were assayed at the beginning and end of 100 cycles to evaluate the changes in the coherence of the CM 5 sensor chip surface after multiple regenerations. The sensitivity of the method was evaluated in terms of LLOQ, which was determined by the criteria that the Biacore response of $\mathrm{C} 225$ at the LLOQ was at least five times that of the blank and the accuracy (relative error) of five replications was within the scope of $80 \%$ to $120 \%$. Intraday precision and accuracy were employed to study the repeatability and reproducibility of the developed method. For intraday variability assessment, three QC samples were analyzed in sextuplicate during a single day; for the interday test, three QC samples were analyzed over 5 consecutive days. The precision was expressed as the $\mathrm{CV}$ relative to the overall mean observed concentration for all analytical runs at each concentration, and the accuracy was determined as the percentage of the overall mean measured concentrations versus the corresponding nominal concentration. The specificity of the assay was validated by adding $\mathrm{C} 225$ at a final concentration of $0.4 \mu \mathrm{g} / \mathrm{ml}$ to $50 \%$ human serum, 50\% Sprague Dawley rat serum, and 20\% pooled monkey serum containing the chimeric recombinant anti-CD20 monoclonal antibody $(0.4 \mu \mathrm{g} / \mathrm{ml})$, human $\gamma$-globulin $(0.4 \mu \mathrm{g} / \mathrm{ml})$, and chimeric recombinant her 2 antibody $(0.4 \mu \mathrm{g} / \mathrm{ml})$, respectively. Each sample was measured in triplicate, and the accuracy was calculated to evaluate the influence of the investigated matrix on the quantitative analysis of $\mathrm{C} 225$.

ELISA-Based Assay for C225 Quantification in Monkey Serum. A 96-well plate was coated with the EGFR diluted with $0.05 \mathrm{M}$ carbonatebicarbonate buffer ( $\mathrm{pH} 9.6$ ) at a protein weight of $2 \mu \mathrm{g} / \mathrm{well}$. Wells without antigen were prepared as the blank controls. The plates were incubated at $4^{\circ} \mathrm{C}$ overnight for antigen adsorption. After removal of the antigen solution by washing with PBST for three times, the wells were treated with $200 \mu \mathrm{l}$ PBS containing $10 \%$ bovine serum for blocking and incubated at room temperature for 2 hours. After washed times with PBST, different concentrations of C225 diluted by $10 \%$ monkey serum were added to the wells (100 $\mu 1 /$ well) and incubated at room temperature for 1 hour. The wells were washed with PBST for six times, and horseradish peroxidase-conjugated goat anti-human IgG diluted by 1:20,000 (100 $\mu \mathrm{l} /$ well) was added as the secondary antibody for incubation at room temperature for 1 hour. Color development was performed with TMB peroxidase substrate $(100 \mu \mathrm{l} /$ well) for 15 minutes, followed by addition of a stopping solution $\left(0.25 \mathrm{~N} \mathrm{H}_{2} \mathrm{SO}_{4}, 100 \mu \mathrm{l} / \mathrm{well}\right)$. The optical density values at $450 \mathrm{~nm}$ were measured, and the final optical density values were derived by subtracting the values for wells without antigen from those for wells with antigen.

Application to Pharmacokinetic Analysis in Rhesus Monkeys. Rhesus monkeys were obtained from the Laboratory Animal Center of the Academy of Military Medical Sciences. The animal room was set at a controlled temperature $\left(25 \pm 1{ }^{\circ} \mathrm{C}\right)$ and humidity $(55 \% \pm 5 \%)$ and a 12-hour light/dark cycle. The animals were fed a standard diet and had free access to water. All experiments were approved by the Institutional Ethical Committee for Care and Use of Laboratory Animals of Academy of Military Medical Sciences and performed in accordance with the Guide for the Care and Use of Laboratory Animals. 
Nine rhesus monkeys (weighing $4.3 \pm 0.7 \mathrm{~kg}$ ) were divided into three equal groups (two males and one female in each group) by a randomization method and received doses of $\mathrm{C} 225(7.5,24$, or $75 \mathrm{mg} / \mathrm{kg})$ via a 30-minute intravenous infusion. The blood samples were collected from the femoral veins of the animals using a puncture needle at $0,0.167,0.333$, and 0.5 hours during the intravenous infusion and at 1, 4, 8, 12, 24, 72, 120, 168, 216, 264, 312, 360, 456, 504, 552, 648 , and 720 hours after dosing. Freshly collected whole blood (approximately $2 \mathrm{ml}$ ) at each time point was collected in a tube and immediately centrifuged ( $4500 \mathrm{~g}$ for 10 minutes at $4^{\circ} \mathrm{C}$ ) for serum separation. All the serum samples were kept at $-80^{\circ} \mathrm{C}$ before analysis. The pharmacokinetic parameters of $\mathrm{C} 225$ were fitted using noncompartmental models by WinNonlin.

\section{Results and Discussion}

\section{Construction of the Au NP-Functionalized SPR Assay for mAb Detection}

SPR sensors have gained increasing attention in the past decade due to the urgent need for fast, reliable, and label-free methods for $\mathrm{mAb}$ detection and quantification in the fields of molecular biology, pharmacology, and other life sciences. However, a major challenge preventing the technology from being successfully translated to pharmacokinetic analysis is its relatively high LLOQ. Au NPs can amplify the SPR signal by altering the SPR angle due to resonance coupling between the surface plasma wave of the Au NP itself and the $\mathrm{Au}$ film on the chip, which further changes the refraction rate on the surface of the chip. Therefore, an Au NP-functionalized SPR assay was developed for the sensitive detection of mAbs, and the procedure is illustrated in Scheme 1. The successful synthesis of Au NPs and goat anti-human IgG-Au NP conjugates was evaluated by transmission electron microscopy and UV spectrophotometry, respectively. The morphologies of the three prepared $\mathrm{Au}$ NP baths were spherically uniform in shape with average diameters of 11.2, 38.7, and $53.1 \mathrm{~nm}$, and no obvious cluster phenomena were observed. After coupling with goat anti-human IgG via electrostatic interactions between the Au NPs and antibodies, the maximum UV absorption wavelengths of the 10,30, and $50 \mathrm{~nm}$ colloidal $\mathrm{Au}$ solutions were shifted from 518 to $527 \mathrm{~nm}$, from 522 to $532 \mathrm{~nm}$, and from 531 to $540 \mathrm{~nm}$ due to protein absorption on the surface of the Au NPs. The results of our work have been published (Shi et al., 2012).

The particle diameter of Au NPs, which is crucial for enhancing the signal responses of C225 in the SPR sensor, was also investigated by injecting goat anti-human IgG-Au NP conjugates prepared with 10, 30, and $50 \mathrm{~nm}$ colloidal Au particles in the manual injection mode. The Au NP-functionalized SPR sensor with $10 \mathrm{~nm}$ colloidal Au particles yielded the highest signal amplification, and the lowest background signal compared with the 30 and $50 \mathrm{~nm}$ colloidal $\mathrm{Au}$ particle-based assays. The results of our work have been published (Shi et al., 2012).

$\mathrm{Au}$ NPs of different sizes resulted in various levels of signal enhancement, as the recognition sites on the antibody were limited, and small $\mathrm{Au}$ NPs possessed the advantage of better surface diffusion (Li et al., 2013). Furthermore, Fernández et al. (2012) reported that small $\mathrm{Au}$ NPs have better diffusion kinetics, favoring their access to the surface (Yuan et al., 2008). The authors also reported that signal enhancement was due to not only Au NPs but also to IgG molecules around the NPs, because more IgG molecules around the NPs increased the overall size. Moreover, IgG molecules around the NPs may also increase the chances of NPs binding to the surface, contributing to the signal enhancement observed. In our study, the number of IgG molecules bound to Au NPs was estimated by the amount of color remaining in the supernatant, as the supernatant was red, whereas the aggregation of NPs was blue. The $10 \mathrm{~nm}$ Au NPs absorbed the most IgG

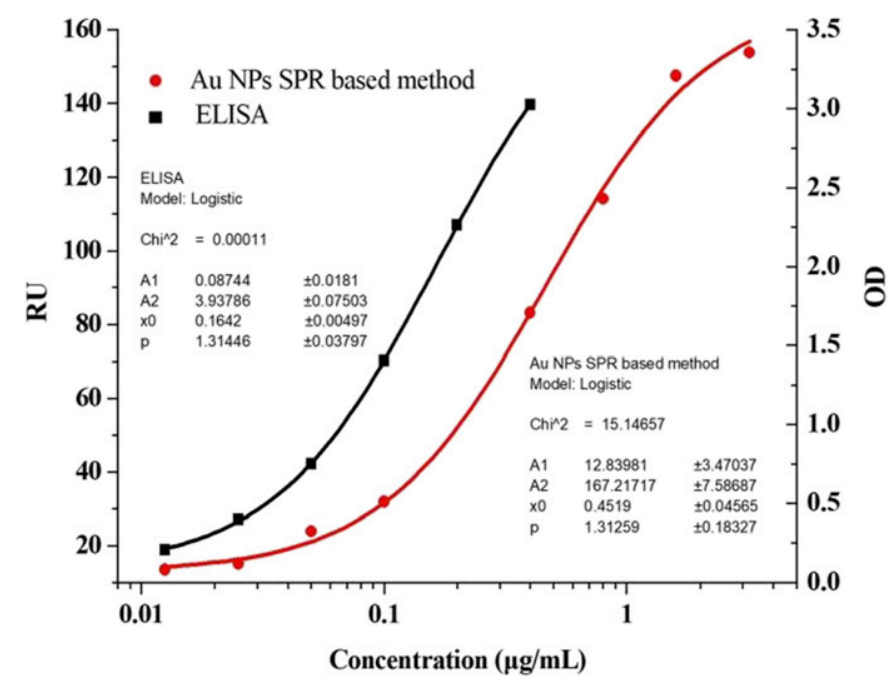

Fig. 1. Standard curve of $\mathrm{C} 225$ quantified by the $\mathrm{Au}$ NP-functionalized surface plasmon resonance assay and ELISA-based method in $20 \%$ monkey serum. The fitted curve was based on the four-parameter sigmoid model (mean \pm S.D., $n=6$ ).

molecules in our study (Shi et al., 2012), which was consistent with the report that NP aggregation results in a significant red shift, changing the solution color from red to blue due to interparticle plasmon coupling (Su et al., 2003; Srivastava et al., 2005).

Therefore, $10 \mathrm{~nm}$ colloidal $\mathrm{Au}$ particles were adopted for functionalization of the SPR sensor and the sensitive detection of trace amounts of mAbs.

\section{Validation of the Au NP-Functionalized SPR Quantitative Assay for $\mathrm{C225}$ in Monkey Serum}

Calibration Curve and LLOQ. In our study, calibration standards were prepared and analyzed at nine different concentrations ranging from 0.0125 to $3.2 \mu \mathrm{g} / \mathrm{ml}$. The calibration curve was constructed by plotting the signals of the Biacore instrument (RU) against the log of the analyte concentrations and then fitted to a four-parameter sigmoid model (Fig. 1). The model fit well to the C225 standard curve over the concentration range. However, the $\mathrm{C} 225$ calibration curve ranged from 0.0125 to $0.4 \mu \mathrm{g} / \mathrm{ml}$ for only the ELISA-based method (Fig. 1).

To determine the LLOQ of the developed assay, C225 at the concentration of $0.0125 \mu \mathrm{g} / \mathrm{ml}$ was assayed five times, and the results are listed in Table 1 . The precision and accuracy of the assay were calculated as $12.60 \%$ and $108.80 \%$, respectively. However, three of the five samples were undetectable at the concentration of $0.00625 \mu \mathrm{g} / \mathrm{ml}$. Therefore, the LLOQ of the assay developed for C225 was defined as $0.0125 \mu \mathrm{g} / \mathrm{ml}$, according to the validation criteria for analytical methods. The LLOQ was four times lower than that of the nanoparticle-free SPR-based assay developed in our previous study $(0.05 \mu \mathrm{g} / \mathrm{ml})$.

TABLE 1

LLOQ evaluation

\begin{tabular}{lccc}
\hline $\begin{array}{c}\text { Nominal Concentration } \\
(\mu \mathrm{g} / \mathrm{ml})\end{array}$ & $\begin{array}{c}\text { Observed Concentration } \\
(\mu \mathrm{g} / \mathrm{ml})\end{array}$ & $\mathrm{CV}(\%)$ & Accuracy (\%) \\
\hline 0.0125 & 0.0117 & 12.60 & 108.80 \\
& 0.0132 & & \\
& 0.0125 & & \\
& 0.0146 & & \\
& 0.0160 & & \\
\hline
\end{tabular}


A

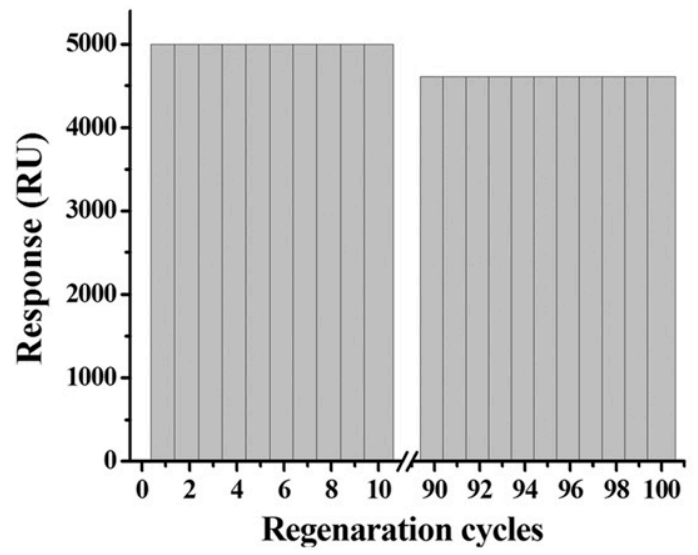

B

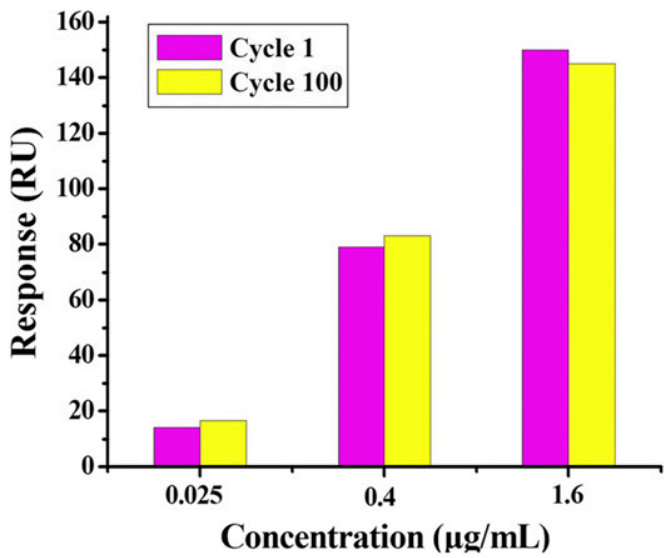

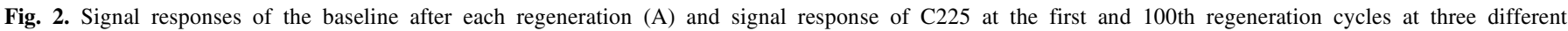
concentrations (B).

Depending on the SPR system, the sensitivity and curve dynamic range reached can be lower than those of microplate-based ELISA methods. In previous works, we proved that the LLOQ of the NP-free SPR-based assay was only $0.05 \mu \mathrm{g} / \mathrm{ml}$ (Che et al., 2009). Improvement of the SPR sensitivity and curve dynamic range can be accomplished by different strategies. For example, the LLOQ can be improved using secondary antibodies (Gobi, 2007; Yuan et al., 2007; Mizuta et al., 2008), and immunoreagents can also be coupled to other elements that increase their mass and refractive index, such as liposomes (Wink et al., 1998), enzymes (Yang and Kang, 2008; Mitchell and Lowe, 2009), polystyrene NPs (He et al., 2004), magnetic NPs (Teramura et al., 2006), and $\mathrm{Au}$ NPs (Lyon et al., 1998; Mitchell et al., 2005; Cao and Sim, 2007). The use of Au NPs has generated substantial interest due to the possibility of controlling their size (Daniel and Astruc, 2004; Boisselier and Astruc, 2009; Li-Na et al., 2010). It has also been postulated that $\mathrm{Au}$ NPs may enhance the sensitivity and curve dynamic range via their own SPR properties (localized surface plasmon resonance), reinforcing the signal by means of electronic coupling of the surface and NP plasmons (Lyon et al., 1998, 1999; Chah et al., 2001).

Methods with better sensitivity and a standard curve dynamic range are good for accurate quantification, as they can detect lower concentrations and require a lower dilution factor. Therefore, the pharmacokinetic parameters from our method were more reliable than SPR without gold nanoparticle.

Baseline Stability. Three QC samples at a high concentration $(1.6 \mu \mathrm{g} / \mathrm{ml})$, a medium concentration $(0.4 \mu \mathrm{g} / \mathrm{ml})$, and a low concentration

TABLE 2

Determination of the precision and accuracy of the intraday assay

\begin{tabular}{lccc}
\hline \multicolumn{3}{c}{ Intraday Assay } & \\
\hline Nominal Concentration of C225 & $\begin{array}{c}0.025 \\
(\mu \mathrm{g} / \mathrm{ml})\end{array}$ & $\begin{array}{c}0.4 \\
(\mu \mathrm{g} / \mathrm{ml})\end{array}$ & $\begin{array}{c}1.6 \\
(\mu \mathrm{g} / \mathrm{ml})\end{array}$ \\
\hline Observed concentration of C225 & 0.020 & 0.413 & 1.801 \\
& 0.028 & 0.392 & 1.862 \\
& 0.024 & 0.392 & 1.671 \\
& 0.021 & 0.397 & 1.586 \\
& 0.025 & 0.428 & 1.603 \\
Mean & 0.027 & 0.380 & 1.541 \\
S.D. & 0.024 & 0.400 & 1.677 \\
Precision $(\%)$ & 0.003 & 0.017 & 0.128 \\
Accuracy $(\%)$ & 13.20 & 4.31 & 7.63 \\
& 96.67 & 100.08 & 104.83 \\
\hline
\end{tabular}

$(0.025 \mu \mathrm{g} / \mathrm{ml})$ were freshly prepared with $20 \%$ rhesus monkey serum and tested in the first and 100th cycles to evaluate alterations in the binding abilities of the CM5 chip to EGFR. Figure 2A shows that EGFR immobilized onto the sensor chip could withstand at least 100 regeneration cycles without significantly changing the baseline (less than $7.8 \%$ ) using $10 \mathrm{mM}$ glycine- $\mathrm{HCl}(\mathrm{pH} 2.5)$, and the changes in the binding capacities of the three different concentrations of $\mathrm{C} 225$ were less than $12 \%$ from the beginning to the completion of the 100 regenerations (Fig. 2B).

Precision and Accuracy. Three QC samples at a high concentration $(1.6 \mu \mathrm{g} / \mathrm{ml})$, medium concentration $(0.4 \mu \mathrm{g} / \mathrm{ml})$, and low concentration $(0.025 \mu \mathrm{g} / \mathrm{ml})$ were used to investigate the intraday and interday precision and accuracy of the developed assay. As shown in Tables 2 and 3 , the precision evaluations with $\mathrm{CV}$ ranged from $4.31 \%$ to $13.20 \%$ (intraday, $n=6$ ) and from $6.75 \%$ to $9.77 \%$ (interday, $n=5$ ). The accuracy ranged from $96.67 \%$ to $104.83 \%$ (intraday, $n=6$ ) and from $96.0 \%$ to $109.26 \%$ (interday, $n=5$ ). These results demonstrate that the developed assay possesses good accuracy and precision for C225 detection in monkey serum.

Specificity of the Assay. The specificity of the assay was determined by comparing the calculated concentrations of C225 in five different matrices with the nominal values. The precision values ranged from $6.25 \%$ to $14.47 \%$, and the accuracy values ranged from $106.0 \%$ to $114.5 \%(n=3)$ in the listed matrix (Table 4$)$. These results indicate that this assay has good specificity and little interference for C225 detection in several types of matrices, which is especially beneficial for quantitatively analyzing C225 in different biologic samples.

TABLE 3

Determination of the precision and accuracy of the interday assay

\begin{tabular}{lccc}
\hline \multicolumn{3}{c}{ Interday Assay } & \\
\hline Nominal Concentration of C225 & $\begin{array}{c}0.025 \\
(\mu \mathrm{g} / \mathrm{ml})\end{array}$ & $\begin{array}{c}0.4 \\
(\mu \mathrm{g} / \mathrm{ml})\end{array}$ & $\begin{array}{c}1.6 \\
(\mu \mathrm{g} / \mathrm{ml})\end{array}$ \\
\hline Observed concentration of C225 & 0.023 & 0.406 & 1.816 \\
& 0.022 & 0.358 & 1.621 \\
& 0.028 & 0.411 & 1.909 \\
& 0.023 & 0.408 & 1.653 \\
Mean & 0.024 & 0.413 & 1.742 \\
S.D. & 0.024 & 0.399 & 1.748 \\
Precision $(\%)$ & 0.002 & 0.023 & 0.118 \\
Accuracy (\%) & 9.77 & 5.81 & 6.75 \\
& 96.0 & 99.8 & 109.26 \\
\hline
\end{tabular}


TABLE 4

Effects of different matrices on the binding of $\mathrm{C} 225$ to the sensor surface (mean \pm S.D., $n=3$ )

\begin{tabular}{|c|c|c|c|}
\hline Concentration of C225 $(0.4 \mu \mathrm{g} / \mathrm{ml})$ & Observed Concentration ( $\mu \mathrm{g} / \mathrm{ml}$, Mean \pm S.D. $)$ & Precision $(\%)$ & Accuracy $(\%)$ \\
\hline $50 \%$ human serum & $0.432 \pm 0.027$ & 6.25 & 108.0 \\
\hline $50 \%$ Sprague Dawley rat serum & $0.442 \pm 0.057$ & 12.93 & 110.5 \\
\hline $20 \%$ monkey serum containing $0.4 \mu \mathrm{g} / \mathrm{ml}$ chimeric recombinant anti-CD20 monoclonal antibody & $0.424 \pm 0.021$ & 4.84 & 106.0 \\
\hline $20 \%$ monkey serum containing $0.4 \mu \mathrm{g} / \mathrm{ml}$ human $\gamma$-globulin & $0.439 \pm 0.064$ & 14.47 & 109.8 \\
\hline $20 \%$ monkey serum containing $0.4 \mu \mathrm{g} / \mathrm{ml}$ chimeric recombinant her 2 antibody & $0.458 \pm 0.040$ & 8.64 & 114.5 \\
\hline
\end{tabular}

In summary, the above results indicate that the newly developed Au NP-functionalized SPR assay displays a wider calibration curve and a higher sensitivity. Therefore, the assay is more suitable for $\mathrm{C} 225$ detection in complex matrices. To the best of our knowledge, this is the first study to report an Au NP-functionalized SPR sensor for mAb quantification using a validated method. Validation experiments show this method to be sensitive, accurate, reproducible, and specific (Table 5).

\section{Pharmacokinetic Study in Rhesus Monkeys}

Pharmacokinetically analyzing $\mathrm{mAbs}$ is challenging because they are usually administered at small dosages during target therapy and need to be monitored for an extended period after dosing. Inspired by the sensitive detection of C225 with the Au NP-functionalized SPR assay, the method was further applied to the pharmacokinetic analysis of C225 in rhesus monkeys following single intravenous infusions of low $(7.5 \mathrm{mg} / \mathrm{kg})$, medium $(24 \mathrm{mg} / \mathrm{kg})$, and high $(75 \mathrm{mg} / \mathrm{kg})$ doses. The serum concentration-time profiles are shown in Fig. 3. The concentrations of C225 in monkey serum after intravenous injection for 504 hours in the low-dose group $(7.5 \mathrm{mg} / \mathrm{kg}$ ) and for 720 hours in the high-dose group $(75 \mathrm{mg} / \mathrm{kg})$ were detected as $0.12 \pm 0.01$ and $4.50 \pm 0.28 \mu \mathrm{g} / \mathrm{ml}(n=3)$, respectively. However, the concentration of C225 was detected at only 456 hours after dosing by our previously established NP-free SPR system (Che et al., 2009). The prolonged monitoring time of C225 in the sera of rhesus monkeys after dosing could contribute to the improved sensitivity of the Au NP-functionalized SPR due to the introduction of Au NPs in the sensor. Therefore, the pharmacokinetic parameters were more reliable than SPR without gold nanoparticle.

Next, the major pharmacokinetic parameters were calculated based on weighted regression analysis. The concentrations of C225 at the same time point increased as the dose increased. The terminal phase elimination half-life $\left(\mathrm{t}_{1 / 2}\right)$ values were $33.15 \pm 1.82,48.03 \pm 0.69$, and $55.72 \pm 0.73$ hours for the three doses, respectively. The $t_{1 / 2}$ was significantly prolonged as the dosage increased. The mean residence time was improved from $105.11 \pm 4.78$ to $152.84 \pm 6.83$ hours, and the area under the curve $(0-t)$ was enhanced from 16,390 \pm 1201.8 to $263,091 \pm 30,698 \mu \mathrm{g} \times \mathrm{h} \times \mathrm{ml}^{-1}$ as the dosage increased. However, the clearance values were $0.459 \pm 0.03,0.466 \pm 0.01$, and $0.29 \pm$ $0.03 \mathrm{ml} \times \mathrm{h}^{-1} \times \mathrm{kg}^{-1}$ for the low-, medium-, and high-dose groups, which decreased as the dosage increased. Therefore, the pharmacokinetics of $\mathrm{C} 225$ was in accordance with the nonlinear model in the investigated dosage range in rhesus monkeys $(7.5-75 \mathrm{mg} / \mathrm{kg})$.

Although there are some reports (Skottrup et al., 2007) on the clinical pharmacokinetic profile of Erbitux (originator of C225), there are no reports on the quantitation of Erbitux in monkey serum or on its preclinical pharmacokinetic parameters.

In our study, use of the ELISA and Au NP SPR methods for the quantitation of $\mathrm{C} 225$ in rhesus monkey serum was validated in accordance with guidelines established by the Bioanalytical Method Validation Guidance for Industry by the Food and Drug Administration and included analyses of the calibration curves, sensitivity, matrix effects, precision, accuracy, and stability. According to the guidelines, SPR, except for ELISA, is the method recommended for ligand-binding analysis. Altlhough both methods were shown to be capable of analyzing the pharmacokinetics of $\mathrm{C} 225$ in rhesus monkeys, the calibration curve of $\mathrm{C} 225$ ranged from 0.0125 to $0.4 \mu \mathrm{g} / \mathrm{ml}$ by the ELISA method and from 0.0125 to $3.2 \mu \mathrm{g} / \mathrm{ml}$ by the Au NP SPR method.

The Au NP-functionalized SPR assay was further applied to the pharmacokinetic analysis of $\mathrm{C} 225$ in rhesus monkeys following single intravenous infusions of low $(7.5 \mathrm{mg} / \mathrm{kg})$, medium $(24 \mathrm{mg} / \mathrm{kg})$, and high $(75 \mathrm{mg} / \mathrm{kg})$ doses, and the major pharmacokinetic parameters were compared with those of the NP-free SPR method (Che et al., 2009). Due to the more sensitive LLOQ of the Au NP-functionalized SPR assay, the time point for detecting $\mathrm{C} 225$ in rhesus monkey serum after dosing was prolonged to 504 hours in the medium-dose group $(24 \mathrm{mg} / \mathrm{kg})$ and to 720 hours in the high-dose group $(75 \mathrm{mg} / \mathrm{kg})$; the detectable time point in the low-dose group $(7.5 \mathrm{mg} / \mathrm{kg})$ was 456 hours for both methods. However, the concentration of C225 was detected at only 456 hours after dosing (low, medium, high dose) by our previously established NP-free SPR system. As a result, the $t_{1 / 2}$ values of C225 in rhesus monkeys obtained by the two methods were not significantly different at the dosage of $7.5 \mathrm{mg} / \mathrm{kg}(P>0.05)$ but were significantly different at dosages of 24 and $75 \mathrm{mg} / \mathrm{kg}(P<0.05)$ due to the higher sensitivity of the Au NP-functionalized SPR assay and the prolonged detection times.

TABLE 5

Pharmacokinetic parameters of C225 in rhesus monkeys after intravenous infusion $(n=3)$

\begin{tabular}{lccc}
\hline & Low Dose $7.5 \mathrm{mg} / \mathrm{kg}$ & Middle Dose $24 \mathrm{mg} / \mathrm{kg}$ & High Dose $75 \mathrm{mg} / \mathrm{kg}$ \\
\hline $\operatorname{AUC}_{(0-\mathrm{t})}\left(\mu \mathrm{g} \times \mathrm{h} \times \mathrm{ml}^{-1}\right)$ & $16,390 \pm 1201.8$ & $51,453 \pm 1199.8$ & $263,091 \pm 30,698$ \\
$\mathrm{AUC}_{(0-\text {-inf })}\left(\mu \mathrm{g} \times \mathrm{h} \times \mathrm{ml}^{-1}\right)$ & $16,396 \pm 1202.7$ & $51,526 \pm 1198.8$ & $26,345 \pm 30,673$ \\
$\mathrm{MRT}(\mathrm{h})$ & $105.11 \pm 4.78$ & $118.89 \pm 1.19$ & $152.84 \pm 6.83$ \\
$\mathrm{CL}\left(\mathrm{ml} \times \mathrm{h}^{-1} \times \mathrm{kg}^{-1}\right)$ & $0.459 \pm 0.03$ & $0.466 \pm 0.01$ & $0.29 \pm 0.03$ \\
$\mathrm{Vss}\left(\mathrm{ml} \times \mathrm{kg}^{-1}\right)$ & $48.26 \pm 4.31$ & $55.39 \pm 0.94$ & $43.85 \pm 4.61$ \\
$\mathrm{t}_{1 / 2}(\mathrm{~h})$ & $33.15 \pm 1.82$ & $48.03 \pm 0.69$ & $55.72 \pm 0.73$ \\
$C_{\max }\left(\mu \mathrm{g} \times \mathrm{ml}^{-1}\right)$ & $160.57 \pm 25.66$ & $1459.5 \pm 52.27$ & $3750.0 \pm 138.42$ \\
\hline
\end{tabular}

AUC, area under the curve; CL, clearance; MRT, mean residence time. 


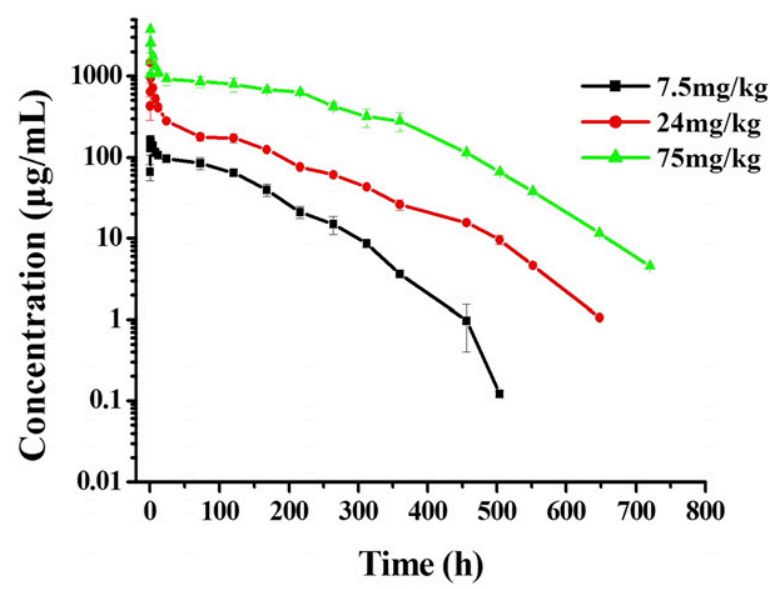

Fig. 3. Serum concentration-time profiles of $\mathrm{C} 225$ in rhesus monkeys during and after intravenous infusion administration at $7.5,24$, and $75 \mathrm{mg} / \mathrm{kg}$. The symbols represent the observed data (mean \pm S.D., $n=3$ ).

The fact that the NP-free SPR-based assay was applied to the pharmacokinetic analysis of Erbitux as well as C225 and that the pharmacokinetic parameters of the two mAbs were not statistically different (Supplemental Fig. 1) proves that the SPR method can be applied to different antibodies. Currently, studies on use of the $\mathrm{Au}$ NP-functionalized SPR assay for analyses of Erbitux as well as other mAbs are ongoing.

\section{Conclusions}

An Au NP-functionalized SPR quantitative assay for the sensitive detection of C225 was developed and validated. The assay displayed a wider calibration curve than the ELISA-based method and had a higher sensitivity than the nanoparticle-free SPR sensor due to the introduction of Au NPs to the SPR sensor, which altered the SPR angle and refraction rate on the surface of the chip via resonance coupling between the surface plasma wave of the Au NP itself and the Au film on the chip. The method was further applied for the pharmacokinetic analysis of C225 in rhesus monkeys in the low-dose $(7.5 \mathrm{mg} / \mathrm{kg})$, middle-dose $(24 \mathrm{mg} / \mathrm{kg})$, and high-dose $(75 \mathrm{mg} / \mathrm{kg})$ groups. The concentration of C225 in monkey serum after intravenous injection for 504 hours in the low-dose group was detectable, and the pharmacokinetics of C225 obtained from weighted regression analysis was accordant with the nonlinear model in the investigated dosage range in rhesus monkeys $(7.5-75 \mathrm{mg} / \mathrm{kg})$. These results indicate that the established method is sensitive, accurate, reproducible, and specific for the detection of mAbs and may have more applications in pharmaco- and toxicokinetic characteristic analyses and clinical evaluations in the future.

\section{Authorship Contributions}

Participated in research design: $\mathrm{X}$. Wang, Che.

Conducted experiments: Bai, Yuan, Che.

Contributed new reagents or analytic tools: X. Wang, Bai, Yuan.

Performed data analysis: X. Wang, Bai, Yuan, Che.

Wrote or contributed to the writing of the manuscript: Bai, Che.

\section{References}

Ben Haddada M, Hu D, Salmain M, Zhang L, Peng C, Wang Y, Liedberg B, and Boujday S (2017) Gold nanoparticle-based localized surface plasmon immunosensor for staphylococcal enterotoxin A (SEA) detection. Anal Bioanal Chem 409:6227-6234.

Boisselier E and Astruc D (2009) Gold nanoparticles in nanomedicine: preparations, imaging, diagnostics, therapies and toxicity. Chem Soc Rev 38:1759-1782.

Cao C and Sim SJ (2007) Signal enhancement of surface plasmon resonance immunoassay using enzyme precipitation-functionalized gold nanoparticles: a femto molar level measurement of anti-glutamic acid decarboxylase antibody. Biosens Bioelectron 22:1874-1880.
Chah S, Hutter E, Roy D, Fendler JH, and Yi J (2001) The effect of substrate metal on 2 -aminoethanethiol and nanoparticle enhanced surface plasmon resonance imaging. Chem Phys 272:127-136.

Che J, Wang H, Chen Z, Li X, Hou Y, Shan C, and Cheng Y (2009) A new approach for pharmacokinetics of single-dose cetuximab in rhesus monkeys by surface plasmon resonance biosensor. J Pharm Biomed Anal 50:183-188.

Chen D, Mei Y, Hu W, and Li CM (2018) Electrochemically enhanced antibody immobilization on polydopamine thin film for sensitive surface plasmon resonance immunoassay. Talanta 182 $470-475$.

Daniel M-C and Astruc D (2004) Gold nanoparticles: assembly, supramolecular chemistry, quantum-size-related properties, and applications toward biology, catalysis, and nanotechnology. Chem Rev 104:293-346.

Fee CJ (2013) Label-free, real-time interaction and adsorption analysis 1: surface plasmon resonance. Methods Mol Biol 996:287-312.

Fernández F, Sánchez-Baeza F, and Marco MP (2012) Nanogold probe enhanced surface plasmon resonance immunosensor for improved detection of antibiotic residues. Biosens Bioelectron 34: $151-158$.

Fischer SK, Yang J, Anand B, Cowan K, Hendricks R, Li J, Nakamura G, and Song A (2012) The assay design used for measurement of therapeutic antibody concentrations can affect pharmacokinetic parameters: case studies. MAbs 4:623-631.

Gurbaxani B, Dostalek M, and Gardner I (2013) Are endosomal trafficking parameters better targets for improving mAb pharmacokinetics than FcRn binding affinity? Mol Immunol 56: $660-674$.

Hage C, Gremse F, Griessinger CM, Maurer A, Hoffmann SHL, Osl F, Pichler BJ, Kiessling F, Scheuer W, and Pöschinger T (2018) Comparison of the accuracy of FMT/CT and PET/MRI for the assessment of antibody biodistribution in squamous cell carcinoma xenografts. $J$ Nucl Med 59:44-50.

Hamilton RG, Saini SS, and MacGlashan D (2012) Surface plasmon resonance analysis of free IgE in allergic patients receiving omalizumab (Xolair). J Immunol Methods 383:54-59.

He L, Smith EA, Natan MJ, and Keating CD (2004) The distance-dependence of colloidal Au-amplified surface plasmon resonance. J Phys Chem B 108:10973-10980.

Hong Y, Jo S, Park J, Park J, and Yang J (2018) High sensitive detection of copper II ions using D-penicillamine-coated gold nanorods based on localized surface plasmon resonance. Nanotechnology 29:215501.

Hossain MZ and Maragos CM (2018) Gold nanoparticle-enhanced multiplexed imaging surface plasmon resonance (iSPR) detection of Fusarium mycotoxins in wheat. Biosens Bioelectron 101: $245-252$.

Jazayeri MH, Amani H, Pourfatollah AA, Avan A, Ferns GA, and Pazoki-Toroudi H (2016) Enhanced detection sensitivity of prostate-specific antigen via PSA-conjugated gold nanoparticles based on localized surface plasmon resonance: GNP-coated anti-PSA/LSPR as a novel approach for the identification of prostate anomalies. Cancer Gene Ther 23:365-369.

Jung I, Kim M, Kwak M, Kim G, Jang M, Kim SM, Park DJ, and Park S (2018) Surface plasmon resonance extension through two-block metal-conducting polymer nanorods. Nat Commun $\mathbf{9}$ 1010

Legeron R, Xuereb F, Chaignepain S, Gadeau AP, Claverol S, Dupuy JW, Djabarouti S, Couffinhal T, Schmitter JM, and Breilh D (2017) A new reliable, transposable and cost-effective assay for absolute quantification of total plasmatic bevacizumab by LC-MS/MS in human plasma comparing two internal standard calibration approaches. J Chromatogr B Analyt Technol Biomed Life Sci 1070:43-53.

Li G, Li X, Yang M, Chen MM, Chen LC, and Xiong XL (2013) A gold nanoparticles enhanced surface plasmon resonance immunosensor for highly sensitive detection of ischemia-modified albumin. Sensors (Basel) 13:12794-12803.

Li H, Ortiz R, Tran L, Hall M, Spahr C, Walker K, Laudemann J, Miller S, Salimi-Moosavi H, and Lee JW (2012) General LC-MS/MS method approach to quantify therapeutic monoclonal antibodies using a common whole antibody internal standard with application to preclinical studies. Anal Chem 84:1267-1273.

Li Y, Monine M, Huang Y, Swann P, Nestorov I, and Lyubarskaya Y (2016) Quantitation and pharmacokinetic modeling of therapeutic antibody quality attributes in human studies. MAbs $\mathbf{8}$ : $1079-1087$.

Li-Na M, Liu D-J, and Wang Z-X (2010) Synthesis and applications of gold nanoparticle probes. Chin J Anal Chem 38:1-7.

Lyon LA, Musick MD, and Natan MJ (1998) Colloidal Au-enhanced surface plasmon resonance immunosensing. Anal Chem 70:5177-5183.

Lyon LA, Peña DJ, and Natan MJ (1999) Surface plasmon resonance of Au colloid-modified Au films: particle size dependence. $J$ Phys Chem B 103:5826-5831.

Mitchell JS and Lowe TE (2009) Ultrasensitive detection of testosterone using conjugate linker technology in a nanoparticle-enhanced surface plasmon resonance biosensor. Biosens Bioelectron 24:2177-2183.

Mitchell JS, Wu Y, Cook CJ, and Main L (2005) Sensitivity enhancement of surface plasmon resonance biosensing of small molecules. Anal Biochem 343:125-135.

Mizuta Y, Onodera T, Singh P, Matsumoto K, Miura N, and Toko K (2008) Development of an oligo(ethylene glycol)-based SPR immunosensor for TNT detection. Biosens Bioelectron 24:191-197.

Neill A, Nowak C, Patel R, Ponniah G, Gonzalez N, Miano D, and Liu H (2015) Characterization of recombinant monoclonal antibody charge variants using OFFGEL fractionation, weak anion exchange chromatography, and mass spectrometry. Anal Chem 87:6204-6211.

Olaru A, Bala C, Jaffrezic-Renault N, and Aboul-Enein HY (2015) Surface plasmon resonance (SPR) biosensors in pharmaceutical analysis. Crit Rev Anal Chem 45:97-105.

Passot C, Desvignes C, Ternant D, Bejan-Angoulvant T, Duveau AC, Gatault P, and Paintaud G (2017) Development and validation of an enzyme-linked immunosorbent assay to measure free eculizumab concentration in serum. Bioanalysis 9:1227-1235.

Piche-Nicholas NM, Avery LB, King AC, Kavosi M, Wang M, O'Hara DM, Tchistiakova L, and Katragadda M (2018) Changes in complementarity-determining regions significantly alter $\mathrm{IgG}$ binding to the neonatal $\mathrm{Fc}$ receptor $(\mathrm{FcRn})$ and pharmacokinetics. MAbs 10:81-94.

Salomon PL and Singh R (2015) Sensitive ELISA method for the measurement of catabolites of antibody-drug conjugates (ADCs) in target cancer cells. Mol Pharm 12:1752-1761.

Shi H, Che J, Chen Z, Liu Y, Shan C, and Cheng Y (2012) Effect of Au NPs of different sizes on sensitivity of SPR-based quantification method. Mil Med Sci 36:280-288.

Singh S, Kumar NK, Dwiwedi P, Charan J, Kaur R, Sidhu P, and Chugh VK (2018) Monoclonal antibodies: a review. Curr Clin Pharmacol 13:85-99. 
Skottrup P, Hearty S, Frøkiaer H, Leonard P, Hejgaard J, O'Kennedy R, Nicolaisen M, and Justesen AF (2007) Detection of fungal spores using a generic surface plasmon resonance immunoassay. Biosens Bioelectron 22:2724-2729.

Srivastava S, Frankamp BL, and Rotello VM (2005) Controlled plasmon resonance of gold nanoparticles self-assembled with PAMAM sendrimers. Chem Mater 17 $487-490$.

Su KH, Wei QH, Zhang X, Mock JJ, Smith DR, and Schultz S (2003) Interparticle coupling effects on plasmon resonances of nanogold particles. Nano Lett 3:1087-1090.

Teramura Y, Arima Y, and Iwata H (2006) Surface plasmon resonance-based highly sensitive immunosensing for brain natriuretic peptide using nanobeads for signal amplification. Anal Biochem 357:208-215.

Then WL, Aguilar MI, and Garnier G (2017) Quantitative detection of weak D antigen variants in blood typing using SPR. Sci Rep 7:1616.

Wang J, Iyer S, Fielder PJ, Davis JD, and Deng R (2016) Projecting human pharmacokinetics of monoclonal antibodies from nonclinical data: comparative evaluation of prediction approaches in early drug development. Biopharm Drug Dispos 37: $51-65$.

Wink T, van Zuilen SJ, Bult A, and van Bennekom WP (1998) Liposome-mediated enhancement of the sensitivity in immunoassays of proteins and peptides in surface plasmon resonance spectrometry. Anal Chem 70:827-832.
Wolf J, Lachmann I, Wagner U, Osman AA, and Mothes T (2011) Quantification of human tissue transglutaminase by a luminescence sandwich enzyme-linked immunosorbent assay. Anal Biochem 419:153-160.

Yang G and Kang SW (2008) Detection of multi-class pesticide residues using surface plasmon resonance based on polyclonal antibody. Food Sci Biotechnol 17:547-552.

Yuan J, Oliver R, Aguilar MI, and Wu Y (2008) Surface plasmon resonance assay for chloramphenicol. Anal Chem 80:8329-8333.

Yuan J, Oliver R, Li J, Lee J, Aguilar M, and Wu Y (2007) Sensitivity enhancement of SPR assay of progesterone based on mixed self-assembled monolayers using nanogold particles. Biosens Bioelectron 23:144-148.

Zhang H, She Z, Su H, Kerman K, and Kraatz H-B (2016) Effects of bipyramidal gold nanoparticles and gold nanorods on the detection of immunoglobulins. Analyst (Lond) 141:6080-6086.

Address correspondence to: Jinjing Che, State Key Laboratory of Toxicology and Medical Countermeasures, Beijing Institute of Pharmacology and Toxicology, No. 27 Taiping Road, Haidian Distrct, Beijing 100085, China. E-mail: chejinjing80@ 126.com 